\title{
Long-range effects on superdiffusive solitons in anharmonic chains
}

\author{
C. Brunhubent and F.G. Mertens \\ Physikalisches Institut, Universität Bayreuth, D-95440 Bayreuth, Germany \\ Y. Gaididei \\ Bogolyubov Institute for Theoretical Physics, 03143 Kiev, Ukraine
}

\begin{abstract}
Studies on thermal diffusion of lattice solitons in Fermi-Pasta-Ulam (FPU)-like lattices were recently generalized to the case of dispersive long-range interactions (LRI) of the Kac-Baker form. The position variance of the soliton shows a stronger than linear time-dependence (superdiffusion) as found earlier for lattice solitons on FPU chains with nearest neighbour interactions (NNI). In contrast to the NNI case where the position variance at moderate soliton velocities has a considerable linear time-dependence (normal diffusion), the solitons with LRI are dominated by a superdiffusive mechanism where the position variance mainly depends quadratic and cubic on time. Since the superdiffusion seems to be generic for nontopological solitons, we want to illuminate the role of the soliton shape on the superdiffusive mechanism. Therefore, we concentrate on a FPU-like lattice with a certain class of power-law long-range interactions where the solitons have algebraic tails instead of exponential tails in the case of FPU-type interactions (with or without Kac-Baker LRI). A collective variable (CV) approach in the continuum approximation of the system leads to stochastic integro-differential equations which can be reduced to Langevin-type equations for the CV position and width. We are able to derive an analytical result for the soliton diffusion which agrees well with the simulations of the discrete system. Despite of structurally similar Langevin systems for the two soliton types, the algebraic solitons reach the superdiffusive long-time limit with a characteristic $t^{1.5}$ time-dependence much faster than exponential solitons. The soliton shape determines the diffusion constant in the long-time limit that is approximately a factor of $\pi$ smaller for algebraic solitons.
\end{abstract}

\section{INTRODUCTION}

Nonlinear excitations like solitons and discrete breathers have been drawing great attention over recent years. They present very robust solutions of nonlinear partial differential equations and nonlinear lattice models which are often used to describe a rather broad set of physical systems [1, 2, 4]. Nonlinear lattices like KleinGordon (KG), Fermi-Pasta-Ulam or the discrete nonlinear Schroedinger (DNLS) lattice (and their counterparts in the continuum approximation) present all to often a strong approximation of the system of interest and it is sometimes not clear to what extend solitons or discrete breathers are also relevant in more realistic models. In the case of biomolecules, the aspiration to highlight their basic functionalities with computer simulations is a challenging task for the computational science nowadays. If we aim to understand principally the role of nonlinear excitations in biomolecules, we have to investigate simpler models than the numerical ab initio calculations. Often used in this context is the Davydov model [5, 6] for energy and charge transport in proteins or the Peyrard-BishopDauxois (PBD) model which describes the melting and the denaturation of DNA [7, 8]. There are convincing evidences that some features of nonlinear excitations in this simplified models are relevant in explaining the functionality of biomolecules. Recently, pump-probe measurements [9] showed that the lifetime of NH stretching

*Electronic address: Christian.Brunhuber@uni-bayreuth.de bands in the model protein ACN is (with about $18 \mathrm{ps)} \mathrm{in}$ good agreement with numerical calculations of the Davydov model [10]. The observation that the PBD model succeeds in determining the thermally induced openings of the DNA strand at functionally relevant sites for the DNA transcription 11 is also an example for the relevance of nonlinear lattice models in biology.

Nowadays, many physicists who work in this field try to extend and improve the basic models in order to make them more realistic. The spatial structure of biomolecules, thermal fluctuations, damping and longrange effects stemming from Coulomb or dipole-dipole interactions have certainly a great influence on the nonlinear excitations and the statistics of the system. It is known that long-range effects can change the features of solitons and discrete breathers qualitatively when the LRI exceed some critical value 12, 13. In FPUlike chains and nonlinear Schrödinger models, they give rise to new types of solitons which can coexist at the same value of the spectral parameter [14]. Controlled switching between such soliton states was recognized as a possible mechanism for energy transport and storage in biomolecules [15]. In the past, nonlinear KG lattices with LRI were frequently studied in investigations of a number of physical phenomena such as dislocations in solids, charge density waves, absorbed layers of atoms or domain walls in ferromagnets and ferroelectrics (see references in [16, 17, 18]). The effect of thermal fluctuations and LRI are usually regarded separately $10,19,20]$ because of their complexity. Nevertheless it is known that LRI can have very interesting effects on the thermodynamics of many different physical systems [21, 22]. 
The Kac-Baker form presents a spatially exponential coupling between different particles which is quite often chosen for the inclusion of LRI in nonlinear lattice models 12, 22, 23]. In the recent publication 24] lattice solitons on FPU-like chain with Kac-Baker LRI in the presence of a thermal reservoir were investigated. In the continuum approximation of the system and with a collective variable (CV) approach, it was possible to derive a Langevin set for the soliton position, similar to the solitons on FPU chains 25]. The solitons on FPU chains (with or without Kac-Baker LRI) show superdiffusive behaviour. The position variance of the soliton shows besides the linear term in the time-dependence also quadratic and cubic terms. In [24], it was demonstrated, that the diffusion mechanism for NNI and for additional LRI are quite similar because the same CV ansatz was used. In the case of Kac-Baker LRI, this approximation is valid unless the soliton velocities do not reach the critical velocity, where the soliton begins to develop a cusp [12].

In order to check the influence of the soliton shape on the diffusion of the soliton, we choose a power-law coupling. This leads in the continuum approximation (CA) to a Benjamin-Ono (BO) equation, which is known to posses algebraic soliton solutions [27]. The first studies in that direction date back to Ishimori [28] who studied anharmonic chains with Lennard-Jones $(2 n, n)$ intermolecular potential and showed that the dynamics is governed by the Benjamin-Ono equation in the case $n=2$ or by the Korteweg-de Vries equation for $n \geq 4$. In the case of cubic and quartic nearest neighbour interactions, a reductive perturbation method yields a $\mathrm{KdV}+\mathrm{mKdV}$ and Benjamin-Ono equation wich was shown to possess exact nonsingular rational solutions [26].

\section{THE MODEL}

Our model is a one-dimensional chain of equally spaced particles of mass $\mathrm{m}(m=1)$ with an interatomic spacing $a(a=1)$. We denote the displacement of the particle $n$ from its equilibrium position as $u_{n}$ (absolute displacement coordinates) and the difference $w_{n}=u_{n+1}-u_{n}$ as relative displacement coordinates. The potential consists of a part $U_{N N}$ similar to the original potential chosen by Fermi, Pasta and Ulam and a long-range potential $U_{L R}$ with a power-law dependence of the harmonic coupling constant $J_{n m}$

$$
\begin{array}{r}
T=\frac{1}{2} \sum_{n=1}^{N}\left(\frac{d u_{n}(t)}{d t}\right)^{2} \\
U_{N N}=\sum_{n=1}^{N} V\left(u_{n+1}(t)-u_{n}(t)\right) \\
V(r)=\frac{r^{2}}{2}-\frac{r^{3}}{3} \\
U_{L R}=\frac{1}{2} \sum_{n} \sum_{m \neq n} \frac{J}{|m-n|^{s}}\left(u_{n}-u_{m}\right)^{2} .
\end{array}
$$

The model covers the physical situation of dipole-dipole $(s=5)$ and Coulomb interactions $(s=3)$ between the particles on the chain, if we restrict ourselves to small relative displacements. The equation of motions in relative displacement coordinates can be obtained from the Lagrangian of the system $L=T-U_{N N}-U_{L R}$

$$
\begin{aligned}
\ddot{w}_{i}(t) & =V^{\prime}\left(w_{i+1}\right)-2 V^{\prime}\left(w_{i}\right)+V^{\prime}\left(w_{i-1}\right) \\
& -J \sum_{m \neq 0} \frac{\left(w_{i}-w_{i+m}\right)}{|m|^{s}} .
\end{aligned}
$$

When we want to consider the effect of a thermal bath, we can add damping and noise terms to the original equation (5) such that they fulfill the fluctuation-dissipation theorem $\left(D^{h y}=2 \nu_{h y} T\right.$ with $k_{B}$ set to unity) [25].

$$
\begin{aligned}
\ddot{w}_{i}(t) & =V^{\prime}\left(w_{i+1}\right)-2 V^{\prime}\left(w_{i}\right)+V^{\prime}\left(w_{i-1}\right) \\
& -J \sum_{m \neq 0} \frac{\left(w_{i}-w_{i+m}\right)}{|m|^{s}}+\nu_{h y}\left(\dot{w}_{i+1}-2 \dot{w}_{i}+\dot{w}_{i-1}\right) \\
& +\sqrt{D^{h y}}\left(\xi_{i+1}(t)-\xi_{i}\right)=0 .
\end{aligned}
$$

We choose hydrodynamical damping which depends on the relative displacement velocities of the particles and presents an intrinsic damping mechanism of the system. The widely used Stokes damping is not appropriate for pulse solitons because one obtains an imaginary dispersion relation the long-wave region of the Fourier spectrum what causes deformations of the soliton [29]. We go to the continuum limit $\left[w_{i}(t) \rightarrow w(x, t), \xi_{i}(t) \rightarrow \xi(x, t)\right.$, $\left.f(x+m, t) \rightarrow e^{m \partial_{x}} f(x, t)\right]$ and find the partial differential equation (PDE)

$$
\begin{aligned}
\partial_{t}^{2} w(x, t) & =2\left(\cosh \left(\partial_{x}\right)-1\right) V^{\prime}(w(x, t)) \\
& -2 J \sum_{m=1}^{\infty} \frac{1-\cosh \left(m \partial_{x}\right)}{m^{s}} w(x, t)+\nu_{h y} \partial_{x}^{2} \partial_{t} w(x, t) \\
& +\sqrt{D^{h y}} \partial_{x}^{2} \xi(x, t) .
\end{aligned}
$$

The further treatment of the system depends on the value of $s$. In [27] it is shown that in the case $s>5$ the CA yields a Boussinesq equation similar to the result without LRI but with a different dispersion parameter. For $3<s \leq 5$ it was proved that the soliton tails are no longer exponential but algebraic and that for $s \leq 3.5$ an energy gap between the soliton states and the plane wave spectra appears. For $s=4$, the equation of motion becomes a Hilbert-Boussinesq equation which can be reduced to the integrable Benjamin-Ono form, which has algebraic soliton solutions.

Since the soliton equation in [24] was of Boussinesq-type and covered the limit of nearest neighbour interactions, we do not expect fundamental new effects for $s>5$. In order to investigate the behaviour of solitons with algebraic tails in the presence of thermal fluctuations, we restrict ourselves to the case $s=4$ where the HilbertBoussinesq equation and the resulting Benjamin-Ono- 
type solitons present a promising system to achieve analytical results. The perturbed Hilbert-Boussinesq equation

$$
\begin{array}{r}
\partial_{t}^{2} w-c^{2} \partial_{x}^{2} w-\lambda \partial_{x}^{4} w-\frac{J \pi}{6} \mathcal{H}\left(\partial_{x}^{3} w\right)+2 \partial_{x}^{2} w^{2}= \\
=\nu_{h y} \partial_{x}^{2} \partial_{t} u+\sqrt{D^{h y}} \partial_{x} \xi(x, t)
\end{array}
$$

with $c^{2}=\left(1+J \pi^{2} / 6\right)$ und $\lambda=(1 / 12-J / 24)$ follows from expanding in (7) the operators for the nearest-neighbour part and for the long-range part $Q\left(s, \partial_{x}\right)=2 \sum_{m}(1-$ $\left.\cosh \left(m \partial_{x}\right)\right) / m^{s}$

$$
2\left(\cosh \left(\partial_{x}\right)-1\right)=2\left(\frac{\partial_{x}^{2}}{2 !}+\frac{\partial_{x}^{4}}{4 !}+\ldots\right)
$$

$$
Q\left(4, \partial_{x}\right)=-\frac{\pi^{2}}{6} \partial_{x}^{2}-\frac{\pi}{6} \mathcal{H}\left(\partial_{x}^{3}\right)+\frac{1}{24} \partial_{x}^{4}
$$

where $\mathcal{H}(f(x))=1 / \pi P \int_{-\infty}^{\infty} f(y) /(y-x) d y$ denotes the Hilbert transform and $\mathrm{P}$ the Cauchy principal value. Similarly like in 24], we will have to rewrite the soliton equation (8) in absolute displacement coordinates in order to find its Lagrangian density (in the case $\nu_{h y}=0$ ). Notice that the $\lambda$-term was neglected in reference [15].

FIG. 1: (Color online) Soliton solutions for power-law $(s=4)$ and Kac-Baker long-range interactions. The two solitons in the left and the right panel each have the same velocity $c_{o}$ and the same energy $H$.

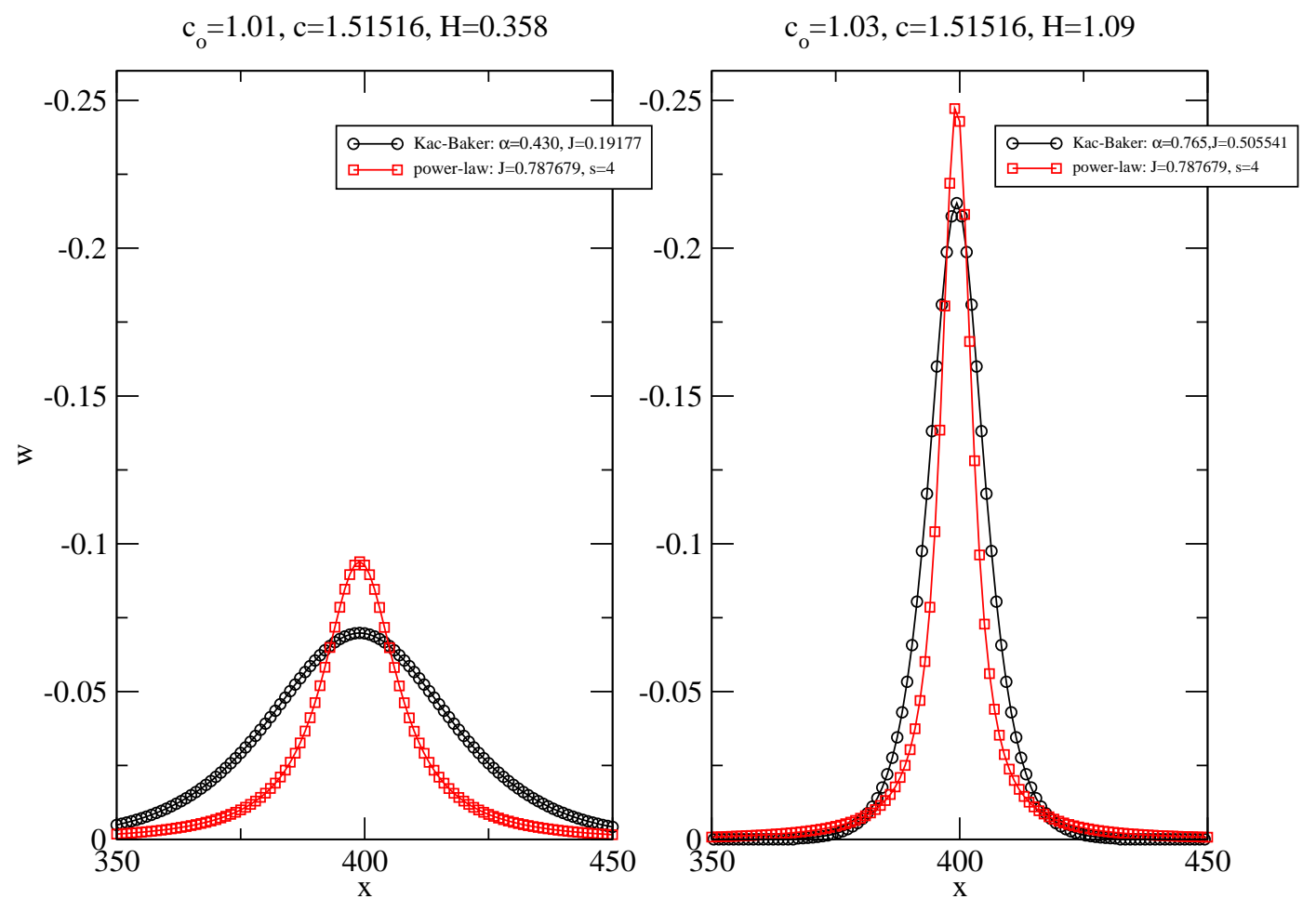

III. COLLECTIVE VARIABLES

The Hilbert-Boussinesq equation in absolute displacement coordinates $u(x, t)$

$$
\partial_{t}^{2} u-c^{2} \partial_{x}^{2} u-\lambda \partial_{x}^{4} u-\frac{J \pi}{6} \mathcal{H}\left(\partial_{x}^{3} u\right)+2 \partial_{x} u \partial_{x x} u=
$$




$$
=\nu_{h y} \partial_{x}^{2} \partial_{t} u+\sqrt{D^{h y}} \partial_{x} \xi(x, t)
$$

can be derived for $\nu^{h y}=0$ from the Lagrangian density

$$
\begin{aligned}
\mathcal{L} & =\frac{u_{t}}{2}-\frac{c^{2} u_{x}^{2}}{2}+\frac{u_{x}^{3}}{J \pi}+\frac{J \pi}{6} u_{x x} \mathcal{H}\left(u_{x}\right)+\lambda \frac{u_{x x}^{2}}{2} \\
& -\sqrt{D^{h y}} u_{x} \xi(x, t) .
\end{aligned}
$$

As a CV ansatz with $X(t)$ and $\sigma(t)$, we will use the Benjamin-Ono-type soliton shape

$$
\begin{gathered}
u(x, t)=-A_{o} \operatorname{Arctan}[\sigma(t)(x-X(t))] \\
w(x, t)=-\frac{A_{o} \sigma(t)}{1+\sigma(t)^{2}(x-X(t))^{2}} .
\end{gathered}
$$

One can check that this ansatz yields the correct values

$$
A_{o}=\frac{J \pi}{3}+4 \lambda \sigma_{o}, \sigma_{o}=\frac{6}{\pi J}\left(v^{2}-c^{2}\right)
$$

(compare with [15]) when one minimizes the action for

$$
\begin{aligned}
L & =\int d x \mathcal{L}= \\
& =\frac{A^{2} \pi}{4}\left(\frac{\dot{\sigma}^{2}}{\sigma^{3}}+\sigma \dot{X}^{2}\right)-\frac{c^{2} A^{2} \pi \sigma}{4}-\frac{\pi}{8} A^{3} \sigma^{2}+\frac{A^{2} J \Pi^{2} \sigma^{2}}{48} \\
& +\frac{A^{2} \pi \gamma \sigma^{3}}{8}+\sqrt{D^{h y}} \int d x \frac{A \sigma}{1+\sigma^{2}(x-X(t))^{2}} \xi(x, t)(15)
\end{aligned}
$$

(with $\lambda=0$ and $\sqrt{D^{h y}}=0$ ) for the soliton parameters $\sigma, A$ and $X(t)$ assuming a coherent excitation with constant velocity $(\dot{\sigma}=\dot{A}=0, \dot{X}=v)$.

To include the damping, we proceed like in [24] with the generalized Hamilton principle of Ostrovsky et al. [30, 31]

$$
\frac{\delta<\mathcal{L}>}{\delta X_{i}}=\frac{\partial<\mathcal{L}>}{\partial X_{i}}-\frac{\partial}{\partial t} \frac{\partial<\mathcal{L}>}{\partial \dot{X}_{i}}=-\left\langle\Phi u_{i}\right\rangle
$$

where $\Phi=\nu^{h y} u_{x x t}$ is a dissipative field and the brackets signify a spatial integration over $x$.

In Fig. 11 we show two solitons with $c_{o}=1.01$ (left panel) and $c_{o}=1.03$ (right panel) on FPU chains with powerlaw LRI $(s=4, J=0.7877, c=1.51516)$ and for the Kac-Baker LRI (described in [24]). The values of $\alpha$ and $J$ for the solitons with the exponential tails in the KacBaker case were chosen to produce a solution with the same velocity and soliton energy $H$. The power-law LRI yield solutions with lorentzian shape (algebraic solitons) whereas the Kac-Baker LRI yield solitons which are well approximated by a sech (exponential solitons).

\section{LANGEVIN SYSTEM}

The eqs. (16) together with the ansatz (13) leads to following stochastic equations for the collective variables $\sigma$ and $X$ :

$$
\begin{array}{r}
\dot{\sigma} \dot{X}=\frac{4 \sqrt{D^{h y}} \sigma^{3}}{A_{o} \Pi} \int d x \frac{\bar{x} \xi(x, t)}{\left(1+\sigma^{2} \bar{x}^{2}\right)^{2}}-\frac{\nu_{h y}}{2} \dot{X} \sigma^{3} \\
\left(\dot{X}^{2}-c^{2}\right)=\frac{A_{o} \sigma}{2}+\frac{\lambda \sigma^{2}}{2}-\frac{8 \sqrt{D^{h y}} \sigma^{2}}{\pi A_{o}} \int d x \frac{\bar{x}^{2} \xi(x, t)}{\left(1+\sigma^{2} \bar{x}^{2}\right)^{2}}
\end{array}
$$

with

$$
A_{o}=\frac{J \pi}{3}+4 \lambda \sigma_{0}, \bar{x}=x-X(t)
$$

In the previous step, we neglected small terms $\sim \dot{\sigma}^{2}, \sim \ddot{X}$ and $\ddot{\gamma}$ in order to obtain technically less comprehensive stochastic equations. The justification of this step was checked by numerical simulations of the complete system and was already successfully applied in the case of KacBaker LRI [24].

We want to rewrite the second equation as an equation for $\dot{X}$. We expand the appearing square root for the small value $\sqrt{D^{h y}}$ to first order and use that the velocity of the damped soliton

$$
v_{d}=\sqrt{c^{2}+\frac{A_{o}}{2} \sigma(t)+\frac{\lambda}{2} \sigma(t)^{2}}
$$

which can be approximated by $v$, the start velocity of the soliton (because $c<v_{d}<v, c \sim v$ ).

We end up in a system of stochastic integro-differential equations for the $\mathrm{CV}$ :

$$
\left(\begin{array}{c}
\dot{\sigma} \\
\dot{X}
\end{array}\right)=\left(\begin{array}{c}
A_{1} \\
A_{2}
\end{array}\right)+\int_{-\infty}^{\infty} d x\left(\begin{array}{cc}
B_{11} & 0 \\
0 & B_{22}
\end{array}\right)\left(\begin{array}{l}
\xi \\
\xi
\end{array}\right),
$$

with

$$
\begin{array}{r}
A_{1}=-\frac{\nu_{h y}}{2} \sigma^{3}, A_{2}=v_{d} \\
B_{11}=\frac{4 \sqrt{D^{h y}} \sigma^{3}}{\pi A_{0} v} \frac{(x-X(t))}{\left(1+\sigma^{2}(x-X(t))^{2}\right)^{2}} \\
B_{22}=-\frac{4 \sqrt{D^{h y}} \sigma^{2}}{\pi A_{0} v} \frac{(x-X(t))^{2}}{\left(1+\sigma^{2}(x-X(t))^{2}\right)^{2}} .
\end{array}
$$

We proceed like in [25, 32] in order to find a statistically equivalent Langevin system to (20) (with the same Fokker-Planck equation in the Stratonovich interpretation 33]) which is more convenient for further numerical and analytical studies. The Langevin-system with two independent Gaussian white noise processes reads:

$$
\left(\begin{array}{c}
\dot{\sigma} \\
\dot{X}
\end{array}\right)=\left(\begin{array}{l}
a_{1} \\
a_{2}
\end{array}\right)+\left(\begin{array}{cc}
b_{11} & 0 \\
0 & b_{22}
\end{array}\right)\left(\begin{array}{l}
\xi^{1} \\
\xi^{2}
\end{array}\right)
$$

with

$$
\begin{array}{r}
a_{1}=-\frac{\nu_{h y} \sigma^{3}}{2}-\frac{D^{h y} \sigma^{2}}{4 A_{o}^{2} \pi v^{2}}, a_{2}=v_{d} \\
b_{11}=\frac{\sqrt{D^{h y} \sigma^{\frac{3}{2}}}}{A_{o} \sqrt{\pi} v}, b_{22}=\frac{\sqrt{D^{h y}}}{\sqrt{\sigma} A_{o} \sqrt{\pi} v} .
\end{array}
$$


The corresponding system in 24] depends similarly on the soliton parameters inverse width, $A_{o}$, velocity $v$ and on the temperature $\sqrt{D}$. Only numerical constants and the velocity $v_{d}$ are different. The broadening of the soliton due to the damping follows the same manner as for exponential solitons in 24, 25]. The result (24) manifests the statement made in 24], that the LRI and the resulting soliton shape determine the velocity $v_{d}(\sigma(t))$ of the damped soliton.

\section{SMALL-NOISE EXPANSION}

We proceed with a small-noise expansion for the parameter $\sqrt{D^{h y}}$ like in [24, 25] in order to derive an ana- lytical expression for the soliton diffusion. The Langevin system (24) is formally very similar to the sytem in 24] for Kac-Baker LRI, only numerical constants and the soliton parameters are different. For algebraic solitons, we can approximate (19) with $v_{d}=c+A_{o} / 4 c \sigma(t)$ because of the smallness of $\lambda$. The analytical solution in this case is technically less extensive and leads practically to the same results. The approximation for $v_{d}$ is not possible in the case of Kac-Baker LRI in 24 because the longrange forces yield a larger term which is quadratic in the inverse soliton width.

The result in the first order of $\sqrt{D^{h y}}$ reads (see Appendix)

$\operatorname{Var}[X(t)]=D^{h y}\left(\frac{A_{o}^{2}[-16(-1+\sqrt{\eta})+(\eta-1)(18-10 \sqrt{\eta}+(\eta-1)(\eta+2))]}{60 A_{o}^{2} c^{2} \nu_{h y}^{3} \pi \sigma_{o}^{3} v^{2} \eta^{\frac{3}{2}}}-\frac{2 c^{2} \nu_{h y}^{2} \eta(-1+\sqrt{\eta}-(\eta-1)(\eta+1))}{3 A_{o}^{2} c^{2} \nu_{h y}^{3} \pi \sigma_{o}^{3} v^{2} \eta^{\frac{3}{2}}}\right)$

with

$$
\eta=1+t_{r}=1+\sigma_{o}^{2} \nu_{h y} t .
$$

The time-dependence of the position variance depends on the time scale $t_{r}$, which explains that the large time limit (and the superdiffusive behaviour) sets in earlier for high-velocity solitons (large values of $\sigma_{o}$ ) for the same damping constant. The corresponding result for KacBaker LRI is even more complex, which stems from the longer expression for $v_{d}$ [24]. The time scale which describes the broadening of the exponential soliton and the diffusion is $t_{r}=1.6 \nu_{h y} \gamma_{o}^{2}$ where $\gamma(t)$ is the inverse width of the exponential soliton

We are interested in the soliton diffusion for large times $t_{r}$. Substituting (27) into (26) and looking for the leading order of $t_{r}$ yields:

$$
\operatorname{Var}[X(t)]_{\text {inf }} \approx \frac{D^{h y}}{60 c^{2} \nu_{h y}^{1.5} \pi v^{2}} t^{1.5},
$$

which differs from the result in 24] only by the numerical constant.

For very small times, the result (26) gives a linear timedependence

$$
\operatorname{Var}[X(t)]_{0}=\frac{4}{3} \frac{D^{h y}}{A_{o}^{2} \pi \sigma_{o}^{2} v^{2}} t .
$$

The results of the small-noise expansion shows the same characteristic dependencies on the soliton parameters as for Kac-Baker LRI in 24]. For intermediate times, the result (26) describes the transition when the position variance turns from the small-time dependence into the longtime limit wherefore a stronger increase in time (mainly $\sim t^{2}$ ) can be observed.

\section{SIMULATIONS}

The simulation of system (6) was performed in the same manner as described in 24] but with power-law long-range coupling instead of the Kac-Baker LRI. The position variance of the soliton was calculated from 100 different realizations of the chain. We used the solitons depicted in Fig. 10 as initial conditions of the chain. The soliton with $c_{o}=1.01\left(\sigma_{o} \approx 0.1\right)$ is much broader than the lattice spacing whereas the soliton with $c_{o}=1.03\left(\sigma_{o} \approx 0.3\right)$ is rather discrete in the soliton center. The coupling parameter is always fixed at the value $J=0.7877$ which yields the sound velocity $c=1.51516$, the same value was used in 24]. In order to compare the results of the Kac-Baker LRI with the power-law LRI and to be able to draw meaningful conclusions, we also use the same damping constant $\nu_{h y}=0.01$ and the same temperature $T=0.0001$ as in Ref. [24].

The analytical result for the position variance (26) agrees rather well with the simulation results (Fig. 22). In general, one expects the analytical results to be slightly smaller than the simulation results because the possible contribution of phonons is not regarded in the calculations of Section III. It was shown for a related short-range model that the phonons have indeed a perceptible influence on the soliton diffusion [35]. The analytical result for the low-velocity soliton diffusion for $c_{o}=1.01$ is too small for small times. Similar discrepancies appeared for Kac-Baker LRI in 24] and they were interpreted as the influence of phonons which cause higher values in the simulations. The analytical result for the high-velocity soliton $c_{o}=1.03$ deviates from the simulation results for 
FIG. 2: (Color online) Position variance of a low-velocity (1.01) and a high-velocity (1.03) soliton on a anharmonic chain with power-law long-range interactions $(J=0.7877$, $s=4)$. We compare the simulation results with equation (26), the result of a small-noise expansion of the Langevin system (24) of the CV theory.
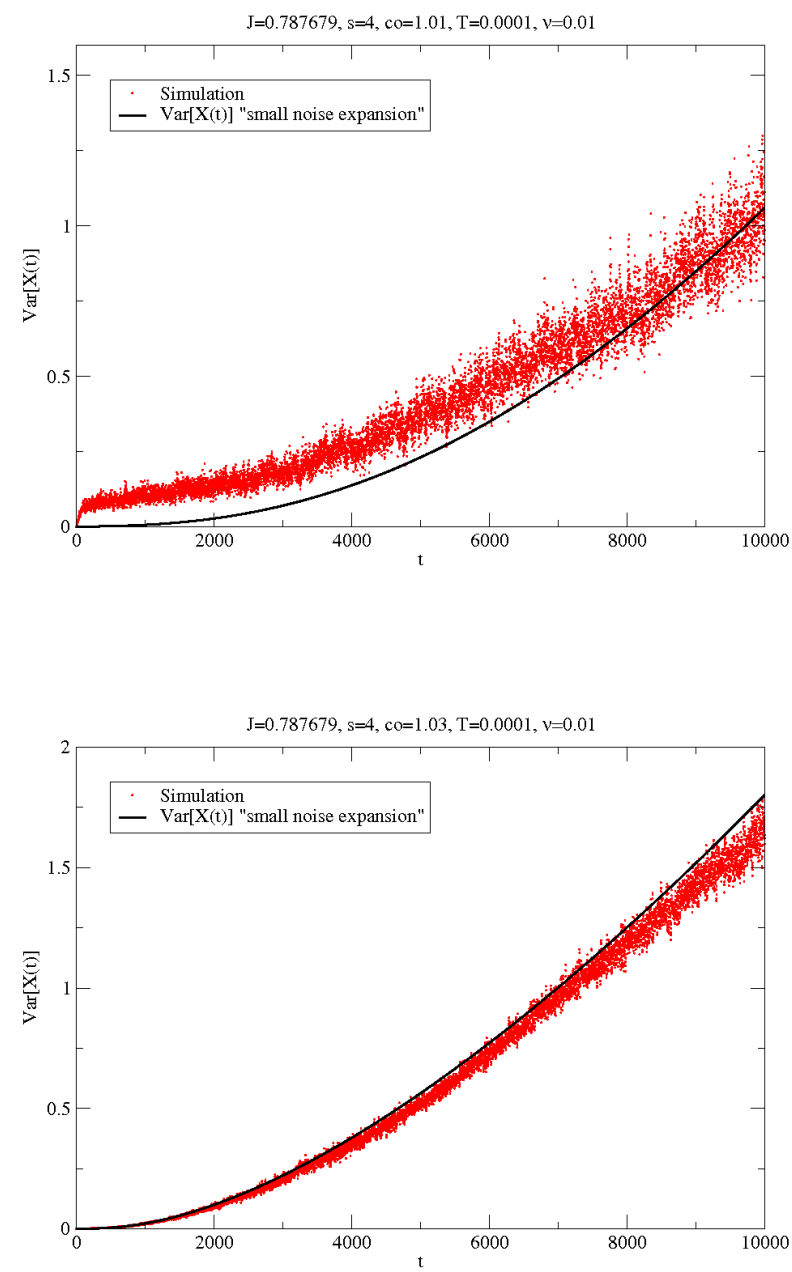

large times. This feature seems to stem from our restriction to first order perturbations in the small-noise expansion. When the solitons are very narrow, the width of the soliton changes quite fast and the first-order correction of $\sigma(t)$ can fail. Such deviations were also observed for FPU chains with or without Kac-Baker LRI [24, 36].

The analytical result for the soliton diffusion depends only on the time scale $t_{r}=\nu_{h y} \sigma_{o}^{2} t$ and for large times the diffusion should approach the result (28) from below. Since the time scale $t_{r}$ depends on the width of the soliton, the long-time approximation for the position variance for high-velocity solitons will be valid for smaller values of $t$ than for low-velocity solitons. For $c_{o}=1.03$ $\left(\sigma_{o} \approx 0.3\right) t_{r}>1$ is valid for $t>1111$ whereas the cor- responding time for $c_{o}=1.01\left(\sigma_{o} \approx 0.1\right)$ is $t>10000$. Therefore, we will check the validity of the long-time approximation result of the position variance for the soliton with $c_{o}=1.03$. In deriving the long-time limit (28) of the position variance, we recognized that the approximation

$$
\operatorname{Var}[X(t)]_{h} \approx \frac{D}{60 c^{2} \nu_{h y}^{2} \pi \sigma_{o}^{3} v^{2}} \frac{t_{r}^{3}}{t_{r}^{1.5}+1.5 \sqrt{t_{r}}}
$$

describes the simulation results very well for all times $t$. Neglecting the $\sqrt{t_{r}}$-term for large $t_{r}$ yields immediately the result (28).

FIG. 3: (Color online) Position variance of a high-velocity soliton $\left(c_{o}=1.03\right)$ on an anharmonic chain with power-law long-range interactions $(J=0.7877, s=4)$. The simulation and two different long-time approximations for (26),

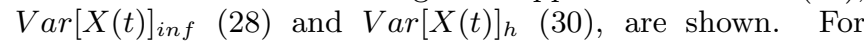
$t=10000\left(t_{r} \approx 9\right)$, the long-time limit is not yet reached, but the simulation results approach this function for long times.

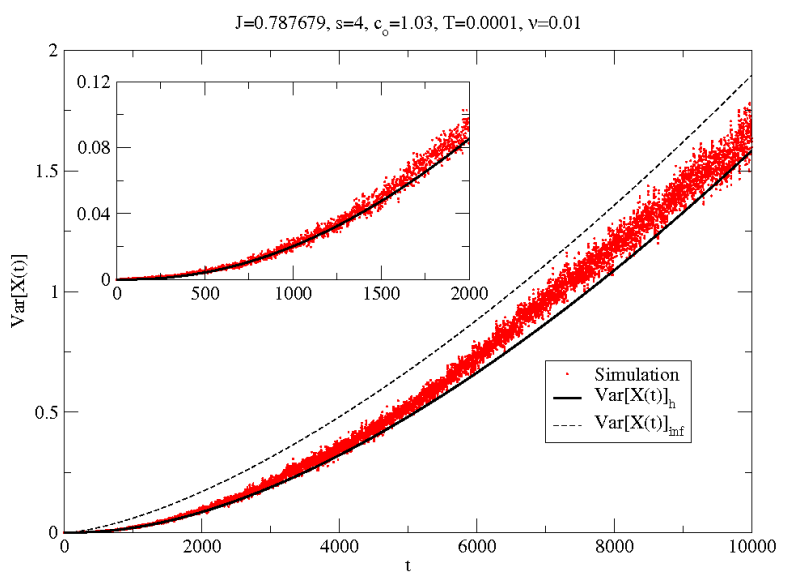

The comparison between the simulation results and $\operatorname{Var}[X(t)]_{h}$ or $\operatorname{Var}[X(t)]_{\text {inf }}$ in Fig. 3] shows that there is practically no linear dependence on $t$ similarly as for high-energy solitons with exponential tails with KacBaker LRI in 24]. The superdiffusion with strongerthan-linear terms in the time-dependence of the position variance is the dominating mechanism for high-energy solitons. The time-dependence of $\operatorname{Var}[X(t)]_{h}$ describes the simulation results very well for all times and the soliton can be described by a time-dependence according to (28) $\left(\sim t^{3 / 2}\right)$ for large times. For lower soliton energies like for $c_{o}=1.01$ (Fig. 2) the normal contributions on $\operatorname{Var}[X(t)]$ are stronger and a comparison with $\operatorname{Var}[X(t)]_{\text {inf }}$ yields a worse agreement because in this case the limit of large $t_{r}$ can not be used for $t<10000$. In the limit of short times $\left(t_{r}<<1\right)$, the quality of the result for the linear contribution in time (29) is very similar to the Kac-Baker case and yields values for $\operatorname{Var}[X(t)]$ which are smaller than the simulation results. For the 
soliton with $c_{o}=1.01$ the slope of (29) is approximately half of the value one would get from a linear fit of the simulation results for small times.

\section{COMPARISON}

Up to now, we have mentioned the similarities between the thermal diffusion of solitons with algebraic and exponential tails on FPU-like chains. Now, we want to demonstrate the main difference, namely the different time-dependence of the superdiffusion in the two cases. We want to directly compare the results for $\operatorname{Var}[X(t)]$ and their time-dependence for two solitons with the same energy and velocity $\left(c_{o}=1.03\right)$ but one on a chain with power-law LRI and the other on a chain with Kac-Baker LRI. For the two solitons, normal diffusion contributions are negligible and the best fits in Fig. 4 demonstrate that the position variance of algebraic solitons increases with $\sim t^{3 / 2}$ whereas for the sech-shaped solitons, the increase goes with a quadratic $\left(\sim t^{2}\right)$ and a cubic $\left(\sim t^{3}\right)$ term. The small-noise expansion results for both soliton types have the same long-time behaviour $\left(\sim t^{3 / 2}\right)$ (compare with [24]) which is reached by the algebraic soliton in Fig. 4 for $t>8000$, whereas the exponential soliton is for times $t<10000$ still in the regime where the longtime limit is not applicable.

The Langevin systems for both soliton types are identical apart from the numerical constants and the values of the soliton parameters like the width. For Kac-Baker LRI, the inverse soliton width $\gamma_{o}=0.132$ at $t=0$ is distinctly smaller than for the power-law case with $\sigma_{o}=0.279$ for the same energy and soliton velocity. Since the time scale $t_{r}$ depends quadratically on the inverse width, it is not surprising that the long-time limit for exponential solitons is valid after much longer times. This argument is decisive for different solitons of the same type. It follows directly from the small-noise expansion that the long-time limit for the algebraic soliton with $c_{o}=1.01$ appears for $\sigma_{o}^{2}\left(c_{o}=1.03\right) / \sigma_{o}^{2}\left(c_{o}=1.01\right) \approx 5.44$ longer times than for the algebraic soliton with $c_{o}=1.03$. In order to estimate when the exponential soliton reaches the long-time asymptotics, we have to concentrate our studies on the discussion of the result $\operatorname{Var}[X(t)]$ from the small-noise expansion for algebraic and exponential solitons (Fig. [5). Simulations for times much longer than $10^{4}$ would be too time-consuming. The double-logarithmic scales in Fig. 5 make clear that the Kac-Baker soliton reaches the long-time limit $\operatorname{Var}[X(t)]_{\text {inf }}$ after much longer times than the power-law soliton. The time window where a stronger time-dependence than $\sim t^{3 / 2}$ appears is approximately ten times larger for the Kac-Baker soliton.

The influence of the soliton shape determines the diffusion constant in $\operatorname{Var}[X(t)]_{\text {inf }}$, which is approximately a factor of $\pi$ larger for exponential solitons. One can equivalently state that the longer transient times to the longtime limit for the exponential soliton is a consequence of
FIG. 4: (Color online) Simulation results for the position variance of two solitons, one on an anharmonic chain with powerlaw long-range interactions $(J=0.7877, s=4)$ and the other soliton on an anharmonic chain with Kac-Baker long-range interactions for $T=0.001$. The interaction radius and the coupling for the Kac-Baker case were chosen in such a way that the two solitons have the same velocity $c_{o}=1.03$ and the same energy. In order to demonstrate the difference in the time-dependence for the two solitons, we added a best fit for the two simulation results for $\operatorname{Var}[X(t)]$. One can clearly see that the position variance of the algebraic soliton approaches $\mathrm{a} \sim t^{1.5}$ time-dependence, whereas in the Kac-Baker case, the time-dependence can be fitted by a quadratic and a cubic term.

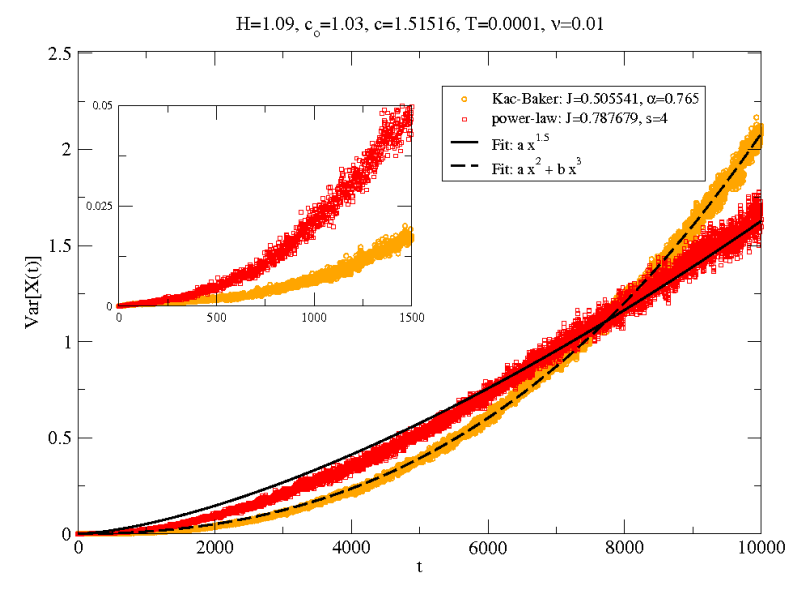

the higher value of the diffusion constant. This remarkable difference for the two soliton types results only from the different soliton shapes because the numerical constant in (28) is determined by the numerical constants in the Langevin system (24) which follow from spatial integrations over the soliton profile in the CV procedure.

The time evolution of the velocity of the energetically equivalent algebraic and exponential soliton, $v_{d}(\sigma(t))$ and $v_{d}(\gamma(t))$, is rather different despite of the same start value and the structural similarities for $v_{d}$. In Fig. 6 we present the simulation results for the mean velocity $\langle\dot{X}(t)\rangle$ of the two solitons in comparison with the zeroth order expressions for $v_{d}$ from the small-noise expansion: $v_{d}\left(\sigma^{(0)}(t)\right)$ and $v_{d}\left(\gamma^{(0)}(t)\right)$ [24].

These hold for the zero temperature case but describe the mean velocity of the two noisy solitons already quite well. We can directly see that the power-law soliton approaches the long-time limit with small soliton velocities earlier than the Kac-Baker soliton. The slower dynamics of the Kac-Baker soliton is caused by its broad, exponential shape and the resulting slower time scale $t_{r}$ which controls the time-evolution of $\gamma^{(0)}(t)$. The dependence of $v_{d}$ on a term $\sim \gamma^{2}$ for exponential solitons (which is negligible for algebraic solitons) influences the soliton velocity (and therefore the superdiffusion), especially for 
FIG. 5: (Color online) $\operatorname{Var}[X(t)]$ and $\operatorname{Var}[X(t)]_{\text {inf }}$ from a small-noise expansion for the two solitons of Fig. 4] The algebraic soliton reaches the long-time limit $\operatorname{Var}[X(t)]_{\text {inf }}$ much sooner than the exponential soliton with the same initial energy $H=1.09$ and velocity $c_{o}=1.03$. The result $\operatorname{Var}[X(t)]_{\text {inf }}$ shows the same time-dependence but the diffusion constant for algebraic solitons is approximately a factor of $\pi$ smaller.

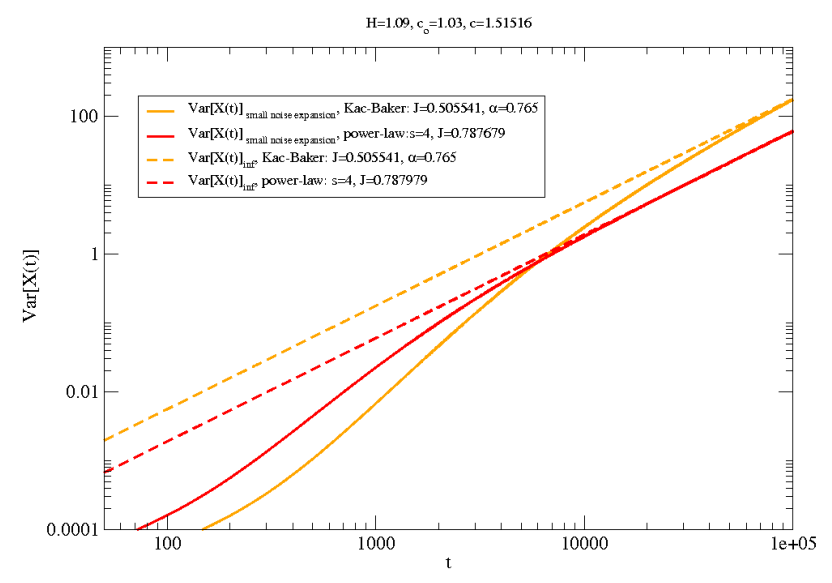

pronounced LRI character and for small times when the long-time limit is not yet reached.

\section{CONCLUSIONS}

We investigated the diffusion of algebraic solitons on FPU-like chains with power-law long-range interactions and derived analytically a result for the position variance which agrees well with the simulations of the discrete system. The results clearly reveal that the soliton diffusion and the damping-induced reshaping of the soliton depends on the time scale $t_{r}=\sigma_{o}^{2} \nu_{h y} t$ which is given by the soliton width and the damping constant. For very broad solitons, the damping effects are quite weak because the particles in the soliton profile have moderate relative velocities and $t_{r}$ evolves slowly in $t$. A small-noise expansion of the Langevin system yields an analytical result for the soliton diffusion which depends on $t_{r}$ and predicts a superdiffusive long-time behaviour $\sim t^{3 / 2}$. This result makes clear why the superdiffusion, which sets in for large $t_{r}$, dominates the diffusion of highenergy solitons $\left(\sigma_{o}^{-1}\right.$ small). These basic features were also found in earlier studies for solitons with exponential shape. The superdiffusion of lattice solitons can therefore be regarded as generic when the lifetime of the solitons is much larger than $1 / \sigma_{o}^{2} \nu_{h y}$. On chains with NNI, this situation can often not be reached for low-energy solitons because they are rapidly destroyed by the fluctuations and the damping. The LRI stabilize the soliton and lead
FIG. 6: (Color online) The simulation results for the mean soliton velocity $\langle\dot{X}\rangle$ in case of a noisy chain with $T=0.0001$ for energetically equivalent algebraic and exponential solitons with $c_{o}=1.03$. The zeroth-order results (without noise) for the soliton velocities $v_{d}\left(\sigma^{(0)}(t)\right)$ and $v_{d}\left(\gamma^{(0)}(t)\right)$ agree rather well with the simulation results and demonstrate the different dynamics of the two soliton types.

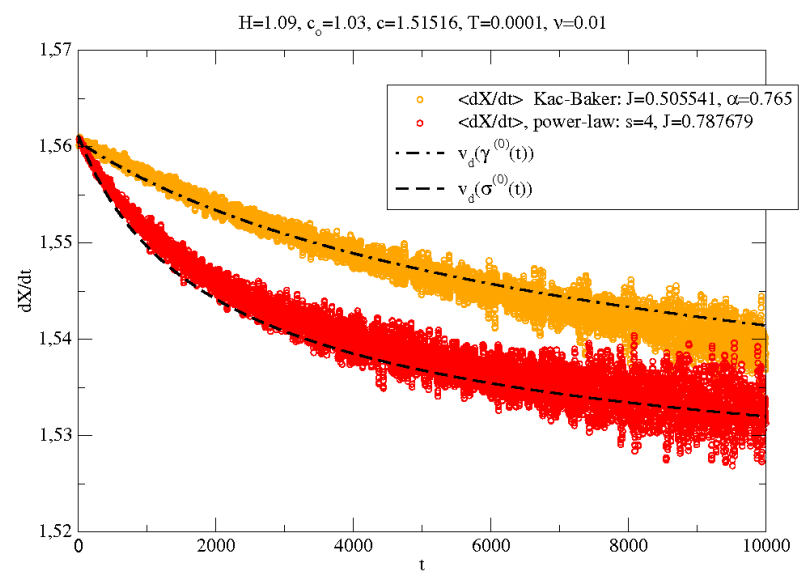

to higher soliton energies and lifetimes which makes the superdiffusion the dominating mechanism.

The shape of the soliton does not change the basics of the diffusion process. The Langevin systems for the collective variabels (inverse width and soliton position) are technically similar, only numerical constants and the dependence of the soliton velocity on the inverse width are different. We proved in simulations and by analyzing the analytical result that despite of the similarities, the superdiffusive behaviour of the two soliton types exhibits striking differences even when the soliton energy and velocity coincide. The algebraic solitons are generally narrower than exponential solitons which leads to faster dynamics for the time scale $t_{r}$ and an earlier validity of the long-time limit with the characteristic $\sim t^{3 / 2}$ time-dependence. The broadness of the exponential solitons, the corresponding slower dynamics for $t_{r}$ and the stronger dependence on higher orders of the inverse width in the soliton velocity shift the $\sim t^{3 / 2}$ region to larger times $t$ which leads to the observed characteristic quadratic and cubic time-dependence in the simulations. The perhaps most remarkable effect of the different soliton shapes is that the exponential solitons show larger position variances than their algebraic counterparts for sufficiently long times. The diffusion constant in the long-time limit is for algebraic solitons approximately a factor of $\pi$ smaller than for exponential solitons. This effect is only caused by the different soliton shapes which determine numerical constants in terms which describe the coupling between the soliton velocity and the fluctuations of the soliton width. Further studies for different 
values of $s$ would be helpful to explain the influence of power-law interactions on the soliton diffusion. Especially the diffusion of solitons on chains with power-law LRI for the case $s<3.5$ could be interesting because a gap between the plane-wave spectra and the soliton energies appears.

\section{APPENDIX: SMALL-NOISE EXPANSION}

The small-noise expansion of the Langevin set (24) follows reference [34] and was already successfully applied to the problem of the diffusion of low-velocity solitons in the FPU system without long-range interactions [25]. We seek an asymptotic solution of the form

$$
\begin{array}{r}
\sigma(t)=\sigma^{(0)}(t)+\epsilon \sigma^{(1)}(t)+\ldots \\
X(t)=X^{(0)}(t)+\epsilon X^{(1)}(t)+\ldots
\end{array}
$$

where $\epsilon$ is a small parameter which is formally introduced to consider the influence of the noise terms as small perturbations $\left(\sqrt{D^{h y}} \sim \epsilon\right)$. The contribution of the drift term in the different orders of $\epsilon$ are calculated following the rule

$$
\begin{aligned}
a_{i}(\sigma(t)) & =a_{i}\left(\sigma^{(0)}+\sum_{m=1}^{\infty} \epsilon^{m} \sigma^{(m)}(t)\right) \\
& =a_{i}^{(0)}(t)+\epsilon \sigma^{(1)}(t) \frac{d a_{i}\left(\sigma^{(0)}(t)\right)}{d \sigma^{(0)}(t)}+\ldots
\end{aligned}
$$

In order to minimize the technical efforts, it is advisable to approximate the velocity $a_{2}=v_{d}$ by the following expression

$$
\begin{aligned}
a_{2} & =\sqrt{c^{2}+\frac{A_{o}}{2} \sigma(t)+\frac{\gamma}{2} \sigma^{2}(t)} \approx c \sqrt{1+\frac{A_{o}}{2 c^{2}} \sigma(t)} \\
& \approx c+\frac{A_{o}}{4 c} \sigma(t)
\end{aligned}
$$

which was checked to be appropriate for typical soliton parameters $J, v$. The equations in the order $\epsilon^{0}$ read:

$$
d \sigma^{(0)}(t)=-\frac{\nu_{h y}}{2} \sigma^{(0)}(t)^{3} d t
$$

$$
d X^{(0)}(t)=\left(c+\frac{A_{0}}{4 c} \sigma(t)\right) d t
$$

These equations describe the damped soliton. The damping induced broadening of the soliton is formally equivalent to the case of exponential soliton solutions in 24]. In the expression for the soliton velocity it is obvious that the damped soliton gets broader (smaller $\sigma$ ) which leads to a slowdown of the soliton. In the limit of long times, the soliton width diverges and the soliton approaches the velocity of sound.

The first order corrections due to the noise read:

$$
\begin{array}{r}
\left.d \sigma^{(1)}(t)=-\sigma^{(1)}(t) \frac{3}{2} \nu_{h y} \sigma^{(0)}(t)^{2}\right) d t+\frac{\sqrt{D} \sigma^{(0)}(t)^{\frac{3}{2}}}{A_{0} \sqrt{\pi} v} d W_{1} \\
d X^{(1)}(t)=\sigma^{(1)}(t) \frac{A_{0}}{4 c} d t+\frac{\sqrt{D}}{\sqrt{\sigma^{(0)}} \sqrt{\pi} A_{0} v} d W_{2}(\mathrm{~A}
\end{array}
$$

If we substitute the result for $\sigma^{(0)}=\sigma_{o} / \sqrt{\nu^{h y} \sigma_{o}^{2} t+1}$ into (A.8) we calculate the first-order expression for the dislocation of the soliton due to the noise

$$
\begin{aligned}
X^{(1)}(t) & =\frac{\sqrt{D} \sigma_{o}^{\left(\frac{3}{2}\right)}}{4 c \sqrt{\pi} v} \int_{0}^{t} d t \eta^{\prime-\frac{3}{2}} \int_{0}^{t^{\prime}} d t^{\prime \prime} \eta^{\prime \prime \frac{3}{4}} d W_{1}\left(t^{\prime \prime}\right) \\
& +\frac{\sqrt{D}}{\sigma_{o} \sqrt{\pi} A_{o} v} \int_{0}^{t} \eta^{\prime \frac{1}{4}} d W_{2}\left(t^{\prime}\right) \\
\eta^{\prime} & =1+\nu_{h y} \sigma_{o}^{2} t^{\prime}
\end{aligned}
$$

After some straightforward calculations we can calculate the position varinace

$$
\left.\operatorname{Var}\left[X_{1}(t)\right]=\lim _{s \rightarrow t}\right\rangle X_{1}(t) X_{1}(s)\langle
$$

which yields the result (26) where we can finally set $\epsilon=$ 1 which is justified if the noise in the system is sufficiently small.
[1] A.C. Scott, Nonlinear Science, University Press Oxford (1999)

[2] AC Scott (editor), The Encyclopedia of Nonlinear Sci4] ence, Routledge, New York, 2005

[4] T. Dauxois, M. Peyrard, Physics of Solitons, Cambridge University Press (2005)

[5] A.S. Davydov, Solitons in Molecular Systems, (Reidel, Dotrecht, 1985)

[6] A.C. Scott, Physics Reports, 217, 1-67 (1992)

[7] M. Peyrard, Nonlinearity 17, R1 (2004)
[8] L. V. Yakushevich, J. Biosci., 26, 305 (2001)

[9] J. Edler, P. Hamm, A.C. Scott, Phys. Rev. Let. 88, 067403 (2002)

[10] L. Cruzeiro-Hansson and S. Takeno, Phys. Rev. E, 56, 894 (1997)

[11] C.H. Choi, G. Kalosakas, K.Ø. Rasmussen, M. Hiromura, A. R. Bishop and A. Usheva, Nucleic Acids Res. 32, 1584

[12] Y.B. Gaididei, N. Flytzanis, A. Neuper, and F.G. Mertens, Physica D 107, 83

[13] S.Flach, Phys. Rev. E 58, R4116 (1998)

[14] P.L. Christiansen, Y.B. Gaididei, F.G. Mertens and S.F. 
Mingaleev, Eur. Phys. J. B 19, 545

[15] S.F. Mingaleev, P.L. Christiansen, Yu.B. Gaididei, M. Johansson and K.Ø. Rasmussen, J. of Biological Physics 25, 41 (1999)

[16] O.M. Braun and Yu. S. Kivshar, Physics Reports 306, 1-108 (1998)

[17] D. Bonart, Phys. Letl A 231, 201-207 (1997)

[18] T. Rössler and J.B. Page, Phys. Rev. E 62, 11460 (2000)

[19] L. Cruzeiro-Hansson, Phys. Lett. A 249, 465 (1998)

[20] K.Ø. Rasmussen, P.L. Christiansen, M. Johansson, Yu. B. Gaididei and S.F. Mingaleev, Physica D 113, 134 (1998)

[21] Dauxois, Ruffo, Arimondo, Wilkens (Eds). Dynamics and Thermodynamics of Systems with Long-Range Interactions, Lecture Notes in Physics 602, Springer (2002)

[22] C. Brunhuber, F.G. Mertens, Y. Gaididei, Phys. Rev. E 73, 016614 (2006)

[23] S.F. Mingaleev, Y.B. Gaididei, and F.G. Mertens, Phys. Rev. E 61, R1044

[24] to be published

[25] E. Arévalo, F. G. Mertens, Y. Gaididei, A. R. Bishop, Phys. Rev. E 67, 016610 (2003)
[26] A.S. Cârstea, D. Grecu, Anca Vişinescu, Phys. Lett. A, 246, 82 (1998)

[27] S.F. Mingaleev, Y.B. Gaididei, and F.G. Mertens, Phys. Rev. E 58, 3833 (1998)

[28] Y. Ishimori, Prog. Theor. Phys. 68, 402 (1982)

[29] E. Arévalo, F. G. Mertens, and A.R. Bishop, Eur. Phys. J. B 2763 (2002)

[30] L.A. Ostrovsky and E.N. Pelinovsky, Sov. Phys. Dokl. 15, 1097 (1971)

[31] A. Jeffrey and T.Kawahara, Asymptotic Methods in Nonlinear Wave Theory, Pitman, London, 1982

[32] C. Brunhuber, F.G. Mertens, Y. Gaididei, Eur. Phys. J. B 42, 103 (2004)

[33] V. Konotop, L. Vázquez, Nonlinear Random Waves (World Scientific, Singapore, 1994)

[34] C.W. Gardiner,Handbook of Stochastic Methods, Springer-Verlag, New York-Heidelberg-Berlin, 1983

[35] F. G. Mertens, E. Arévalo, and A.R. Bishop, Phys. Rev. E 72, 036617 (2005)

[36] E. Arévalo, Dissertation, Universitaet Bayreuth (2004) 